\title{
COMPARISON THEOREMS FOR A GENERALIZED MODULUS OF CONTINUITY
}

\author{
BY JAN BOMAN AND HAROLD S. SHAPIRO
}

Communicated by Ralph Boas, June 2, 1969

1. Notation and definitions. Let $R^{m}$ denote real Euclidean $m$-space. We shall employ standard vector notations, whereby $t=\left(t_{1}, \cdots, t_{m}\right)$, $u=\left(u_{1}, \cdots, u_{m}\right)$ denote points of $R^{m}, t u=\sum_{1}^{m} t_{i} u_{i}$ and $|t|=(t t)^{1 / 2}$. In connection with Fourier transforms $x=\left(x_{1}, \cdots, x_{m}\right)$ denotes a point of a "dual" copy of $R^{m} . M=M\left(R^{m}\right)$ shall denote the totality of bounded complex-valued Borel measures on $R^{m}$, made into a Banach algebra in the usual way, i.e. the "product" of the measures $\sigma, \tau$ is the convolution $\sigma * \tau$ and the norm of a measure is its total variation. $\hat{\sigma}$ shall denote the Fourier transform of $\sigma$, and $W=W\left(\hat{R}^{m}\right)$ the Banach algebra of Fourier transforms of elements of $M$. In $W$ the "multiplication" is ordinary point-wise multiplication of functions. $W$ is isometrically isomorphic to $M$ under the map $\sigma \rightarrow \hat{\sigma}$.

For $f \in L^{p}\left(R^{m}\right)$ with $1 \leqq p \leqq \infty$ and $\sigma \in M$ we write $f * \sigma$ to denote the function $g$ such that $g(t)=\int f(t-u) d \sigma(u)$. (This is defined for almost all $t$; moreover $g \in L^{p}$.) For $a>0$ let $\sigma_{(a)}$ denote the measure defined by $\sigma_{(a)}(E)=\sigma\left(a^{-1} E\right)$ for all Borel sets $E$. We have $\left\|\sigma_{(a)}\right\|=\|\sigma\|$.

For $f \in L^{p}, \sigma \in M$ and $a>0$ let us define

$$
\begin{aligned}
& D_{\sigma . p}(f ; a)=\left\|f * \sigma_{(a)}\right\|_{p}, \\
& \omega_{\sigma, p}(f ; a)=\sup _{0<b \leqq a} D_{\sigma, p}(f ; b) .
\end{aligned}
$$

We shall call $\omega_{\sigma, p}$ (for fixed $f$, considered as a function of $a$ ) the $\sigma, p$ modulus of $f$. The reason for the choice of this term is that when $m=1$ and $\sigma$ is specialized to be a "dipole measure" with "masses" of +1 at $t=0$ and -1 at $t=1$ we obtain the usual $L^{p}$ modulus of continuity. Various other specializations of $\sigma$ lead to "moduli" that are of interest in studying the smoothness and approximation properties of functions (see [1], [2]).

We shall say a measure $\sigma$ satisfies the Tauberian condition if $\hat{\sigma}(x)$ does not vanish at all points of any half-ray through the origin, i.e. if given $x,|x|=1$ there is a positive number $a$ such that $\hat{\sigma}(a x) \neq 0$.

\section{The comparison theorems.}

Theorem 1. Suppose $\sigma, \tau \in M\left(R^{m}\right), \sigma$ satisfies the Tauberian condition, and there is a function $F \in W$ such that $\hat{\tau}(x)=\hat{\sigma}(x) F(x)$ in some neighborhood of the origin. Then for $f \in L^{p}(1 \leqq p \leqq \infty)$ and $a>0$ 


$$
\omega_{r, p}(f ; a)^{\bar{p}} \leqq A \int_{0}^{B a} \omega_{\sigma, p}(f ; v)^{\bar{p}} \frac{d v}{v}
$$

where $A, B$ depend only on $\sigma, \tau$ and $p$, and

$$
\begin{aligned}
p & =\min (p, 2), & & p<\infty, \\
& =1, & & p=\infty .
\end{aligned}
$$

Theorem 2. Suppose $\sigma, \tau \in M\left(R^{m}\right)$ and $\sigma$ satisfies the Tauberian condition. Suppose there exists a function $P$ which is positive-homogeneous of degree $r>0$ (i.e. $P(b x)=b^{r} P(x)$ for $b \geqq 0$ ), and functions $F, G \in W$ such that in some neighborhood of the origin, $P(x)=G(x)$ and $\hat{\tau}(x)=\hat{\sigma}(x) F(x)$. Then, for $f \in L^{p}(1 \leqq p \leqq \infty)$ and $a>0$

$$
\omega_{\tau, p}(f ; a)^{\bar{p}} \leqq A \int_{0}^{\infty}\left[\min \left(1,(a / v)^{r}\right) \omega_{\sigma, p}(f ; B v)\right]^{\bar{p}} \frac{d v}{v}
$$

where $A, B$ depend only on $\sigma, \tau$ and $p$, and $\bar{p}$ is given by (2).

This theorem, suitably specialized, yields a large portion of the known theorems, both "direct" and "inverse," concerning the approximation of functions by trigonometric polynomials and by convolution integrals, as well as sharp comparison theorems for "moduli of smoothness" of different orders.

\section{An "embedding theorem."}

Theorem 3. Suppose $\sigma \in M\left(R^{m}\right)$ satisfies the Tauberian condition and $s$ is a positive integer. Let $1 \leqq p \leqq \infty$, and suppose $f \in L^{p}$ and

$$
\int_{0}^{1}\left[a^{-s} \omega_{\sigma, p}(f ; a)\right]^{\bar{p}} \frac{d a}{a}<\infty
$$

where $\bar{p}$ is defined by (2). Let $\alpha$ denote any multi-index $\left(\alpha_{1}, \cdots, \alpha_{m}\right)$ with $\Sigma \alpha_{i} \leqq s$. Then the (distributional) derivative $D^{\alpha}$ f is a function which is locally of class $L^{p}$. In particular, if $f$ has compact support it belongs to the Sobolev space $W_{p}^{s}$.

For instance, specializing to $m=1$, and $\sigma$ having "masses" of $+1,-2,+1$ at $t=0,1,2$ respectively, we obtain the Weiss-Zygmund Theorem [3] that a function whose (second-order) $L^{p}$ modulus of smoothness is $O\left(a(\log (1 / a))^{-c}\right)$ with $c \bar{p}>1$ is absolutely continuous and its derivative belongs locally to $L^{p}$.

4. Concluding remarks. The above theorems can be generalized (as in Theorem 2 of [2]) to allow several measures $\sigma_{1}, \cdots, \sigma_{r}$ in place 
of $\sigma$ in the hypotheses. They also extend mutatis mutandis to the standard $L^{p}$ spaces of periodic functions.

Details and applications will be given elsewhere. Here we remark only that, except for $p=1$ and $p=\infty$, the above results are essentially stronger than those indicated in [2], and are in various senses "best possible." The proofs are all based on a generalized version of the Littlewood-Paley inequality for trigonometric series.

\section{REFERENCES}

1. H. S. Shapiro, Smoothing and approximation of functions, Van Nostrand, Princeton, N. J., 1969.

2. - A Tauberian theorem related to approximation theory, Acta Math. 120 (1968), 279-292.

3. M. Weiss and A. Zygmund, A note on smooth functions, Indag. Math. 21 (1959), 52-58.

University of Stockholm, Stockholm, SWeden AND

University of Michigan, ANn Arbor, Michigan 48104 